\title{
Dressing monitoring with a digital signal processor
}

\author{
R. F. Luzia ${ }^{1}$, P. R. de Aguiar $^{1}$, E. C. Bianchi ${ }^{2} \&$ M. M. Spadotto ${ }^{1}$ \\ ${ }^{I}$ Department of Electrical Engineering, UNESP - Univ Estadual Paulista, \\ Brazil \\ ${ }^{2}$ Department of Mechanical Engineering, \\ UNESP - Univ Estadual Paulista, Brazil
}

\begin{abstract}
Grinding is a finishing process in machining operations, and the topology of the grinding tool is responsible for producing the desired result on the surface of the machined material. The tool topology is modeled in the dressing process and precision is therefore extremely important. This study presents a solution in the monitoring of the dressing process, using a digital signal processor (DSP) operating in real time to detect the optimal dressing moment. To confirm the monitoring efficiency by DSP, the results were compared with those of a data acquisition system (DAQ) and offline processing. The method employed here consisted of analyzing the acoustic emission and electrical power signal by applying the DPO and DPKS parameters. The analysis of the results allowed us to conclude that the application of the DPO and DPKS parameters can be substituted by processing of the mean acoustic emission signal, thus reducing the computational effort.
\end{abstract}

Keywords: data acquisition, digital signal processor, dressing operation, grinding process.

\section{Introduction}

Grinding is a workpiece finishing process that accounts for approximately $25 \%$ of all machining processes. Because this operation is usually the last one in the manufacturing sequence, all the time and quality-related problems generated along the chain of processes involved in the fabrication of a component must be 
solved during grinding. As a result, any benefit obtained in grinding is of high value to the entire process [1].

Continuous grinding causes the grinding wheel to undergo surface wear and assume an inadequate shape, causing the appearance of dull edges, i.e., loss of cutting edges, as well as clogging of its pores by chips, making the removal of new material inefficient [2].

This grinding wheel surface wear reduces the tool's cutting ability, which in turn increases the temperature of the grinding process due to interaction between the cutting energy and the workpiece surface. Increases in temperature may lead to thermal damage, which may vary from a superficial change in color to fracture of the workpiece [3].

Analyzing the operating conditions and the state of the cutting tool enables one to predict the tool's service life. However, monitoring of the grinding process is highly complex due to the large number of parameters involved [4].

Focusing on the need to monitor and improve the grinding process, Webster et al. [5] studied the emission of acoustic signals from the workpiece surface and found that the so-called raw signal (AEraw) reliably represents the existence of faults on the surface of the workpiece and grinding wheel. Based on these studies, it was also concluded that the effective acoustic emission signal (AErms) can be used in the process for the same purpose, but, according to the integration time constant, some important data may be lost due to the characteristic of low pass filtration in this type of operation.

Aguiar et al. [6] proposes a combination of the effective acoustic emission signal and the mean electrical power signal of the grinding wheel motor, in a statistical tool called DPO to detect the occurrence of burn during the grinding process. By means of a second statistic studied by Dotto et al. [7], called DPKS, it is possible to identify the beginning and end of burn in most cases. This statistic also uses the same signals as DPO, and was designed to present the highest possible sensitivity in the process.

Because the effect of burn on the workpiece results from the wear of the grinding wheel surface, the occurrence of this effect can be reduced in the preparation of the grinding wheel prior to the grinding. The dressing operation prepares the grinding wheel, restoring the cutting edges and removing the chips from the grinding wheel, thereby reducing the temperature at the point of contact between the workpiece and the grinding wheel and improving the grinding process. When correct dressing parameters and conditions are used, it is possible to increase the ool's service life [3].

Aiming to improve the dressing process, Hellmeister [8] proposes the use of the raw acoustic emission signal in this process and concludes that by analyzing this signal it is possible to detect faults on the grinding wheel surface during dressing, which is in agreement with what Webster et al. [5] found in the grinding process.

Aguiar et al. [9] proposed the use of DPO and DPKS statistics in the dressing process, both of which presented satisfactory results. An analysis of the results of the statistics allowed for the determination of the moment when dressing could be concluded. 
This article proposes the implementation of data acquisition and DPO and DPKS statistics processing routines on DSP hardware to ascertain the possibility and feasibility of developing a device operating in real time to monitor the dressing process.

After carrying out tests and analyzing the data, it was found that the DSP system showed satisfactory results, and that it is feasible to develop a tool based on this type of processing to monitor the dressing process. Moreover it was found that for the conditions applied, the calculated statistics can be substituted for the mean effective acoustic emission signal, which reduces the computational effort and simplifies the hardware.

\section{Monitoring of the dressing process}

The topology of the grinding wheel is defined by the spatial distribution and shape of the abrasive grains on the surface of the tool. Before beginning the grinding process, the grinding wheel must be prepared, i.e., material must be removed from the cutting surface with the least possible macroscopic alteration, and the dressing stage consists of conditioning the grinding wheel with the purpose of imposing a given grinding behavior. In conventional abrasive grinding wheels, these stages are carried out by the same process, which is normally called dressing [10]. In general, the dressing operation reconditions the surface of the grinding wheel so as to recover its cutting edges, which have been deteriorated by use, removing chips and allowing for the removal of new material [11].

According to Marinescu et al. [12], the process of preparing the grinding wheel is very important because the tool's topology is directly associated with its grinding performance through the characteristics of the forces acting upon the system, i.e., power consumed, process temperature, and the final surface of the ground workpiece. The choice of the values of the parameters to be used in the dressing process depends on the desired characteristics and on the desired final result of the grinding process; hence, the dressing is defined by the effect of grinding.

In vitrified conventional abrasive grinding wheels, the dressing tool is called a dresser. The most common dressers are single or multiple-tip diamond dressers with transverse movement $[12,13]$. Dresser diamonds may be synthetic or natural and are available in several shapes and configurations, with some of their specification parameters being the type and way they are mounted in the tool, and the crystal's number, size and geometric characteristics.

The action of the dresser is similar to that of a turning tool and the volume of abrasive material removed from the grinding wheel in each pass is proportional to the dressing depth $\left(a_{d}\right)$ [12]. The advancement of the dressing tool across the rotating grinding wheel causes the formation of a screw whose step is called a dressing step $\left(f_{d}\right)$, which is given by:

$$
f_{d}=\pi \cdot d_{s} \cdot v_{f d} / v_{s}
$$


where $d_{s}$ is the initial diameter of the grinding wheel, $v_{f d}$ is the transverse advancement speed of the dresser, and $v_{s}$ is the tangential speed of rotation of the grinding wheel.

Linked to parameter $a_{d}$ is the width of action of the dresser $\left(b_{d}\right)$, which defined the width of contact between the dresser and the grinding wheel [12]. Although the efficiency and quality of the dressing process depend mainly on $a_{d}$ and $f_{d}$, the most important parameter is the overlap factor $\left(U_{d}\right)$, which expresses a relationship between $b_{d}$ and $f_{d}$.

$$
U_{d}=b_{d} / f_{d}
$$

The adjustment of parameters $a_{d}$ and $f_{d}$ defines the type of dressing [12]. Fine dressing is when both these values are low, and in this type of dressing, the distance between grains is small and grain density on the surface is high, causing the volume of material removed in grinding to be greater, with high initial forces and power that decrease with the wear of the grinding wheel.

Analogously, coarse dressing is when $a_{d}$ and $f_{d}$ have high values. Contrary to fine dressing, coarse dressing increases the distance between the abrasive grains, reducing the density, which in turn reduces the forces and power, although the roughness of the ground surface is greater.

Köning apud Aguiar [9] report that due to the wear that occurs at the tip of the dresser, adjustment of the process based solely on $a_{d}$ and $f_{d}$ is inadequate, since it does not consider the width of action, which varies with use. Therefore, the $U_{d}$ parameter is more suitable for the adjustment of the dressing process, because it takes into account the real width of action according to the wear at the tip, making the process more efficient.

According to Xue apud Aguiar [9], the number of dressing passes depends on the intensity of wear of the grinding wheel, the grain size, and the depth of wear to be removed. Normally two or three successive passes eliminate the effects of previous dressing and grinding. However, in the dressing process errors may occur that affect the quality of the preparation of the grinding wheel in different ways. These errors may result from variations in dressing depth, dresser wear and other problems. Thus, a monitoring system for the process may detect such errors, as well as determine the moment when dressing should be concluded.

Xue et al. [22] use the acoustic emission signal to monitor the dressing process online in order to detect grinding wheel deformations and contour errors. This signal has proved efficient in the identification of faults that may occur in the process. Also, according to Xue et al., monitoring the dressing parameters results in a more homogeneous surface roughness and online monitoring can reveal the dressing depth behavior.

Due to the importance of the grinding process, numerous studies have been carried out to improve it. For example, acoustic emission sensors have been utilized to detect the contact between the grinding wheel and the dresser (Oliveira et al. [14]; Karpuschewski et al. [4]), monitor the dressing depth (Inasaki [15]), monitor the dresser lead (Inasaki [15]; Webster et al. [16]), diagnose dressing failures (Karpuschewski et al. [4]; Konig and Meyen [17]; Kwak and Ha [18]; Lee et al. [19]), model the dressing process (Linke [20]), 
finish dressing (Aguiar et al. [9]), and a method without contact has been proposed (Young and Chen [21]).

Because it is not possible to determine the number of passes required to complete the dressing process, Aguiar et al. [5] use DPO and DPKS statistics to monitor the process and determine the optimal dressing moment, i.e., when the process can be concluded. These statistics are used in the detection of the occurrence of the burn phenomenon in the grinding process.

According to studies carried out by Aguiar et al. [6], the combination of the effective acoustic emission signals $\left(\mathrm{AE}_{\mathrm{rms}}\right)$ and the mean electrical power provide an excellent indicator of the occurrence of burn. This combination gave rise to the DPO parameter, which is given by the multiplication of the standard deviation of the acoustic emission signal by the maximum value of the mean electrical power signal recorded in the pass.

$$
D P O=\sigma_{E A_{r m s}} \cdot M A X(\bar{P})
$$

where $\sigma_{E A r m s}$ is the standard deviation of the effective acoustic emission signal in the pass and $M A X(P)$ is the maximum mean electrical power in the pass.

Modifying the expression of the DPO parameter in order to achieve greater sensitivity, the DPKS parameter was developed, which is given by multiplying the standard deviation of $\mathrm{AE}_{\mathrm{rms}}$ by the sum of the difference between the instantaneous value of the mean electrical power and the standard deviation of this power raised to the $4^{\text {th }}$ power [7].

$$
D P K S=\sigma_{E A_{m n s}} \cdot \sum_{i=1}^{i=m}\left(\bar{P}_{i}-\sigma_{\bar{P}}\right)^{4}
$$

where $\sigma_{P}$ is the standard deviation of the mean electrical power signal in the pass, and $P_{i}$ is the instantaneous value of the mean electrical power.

\section{Methodology}

This study consisted of analyzing the possibility of implementing a monitoring system for the dressing process using a digital signal processor (DSP) model ADSP-BF533 of Analog Devices' BlackFin family operating in real time. The analysis was performed by comparing the processing results of the DSP system in real time against the offline processing results in routines executed with MATLAB software. Os signals used in the MATLAB processing were obtained by a data acquisition system (DAQ), using LabVIEW software and a National Instruments signal acquisition board.

The DSP system was programmed to capture the mean quadratic acoustic emission signal (AERMS) and the mean electrical power $(\mathrm{P})$ of the grinding wheel motor by means of a digital-to-analog converter. The acoustic emission signal was obtained by a passive response sensor at a frequency of $50 \mathrm{~Hz}$ to 1 $\mathrm{MHz}$ and preprocessed by a DM-42 acoustic emission module. The acoustic emission module receives the acoustic emission signal from the sensor and processes the RMS value with a time constant of $1 \mathrm{~ms}$. To prevent saturation and 


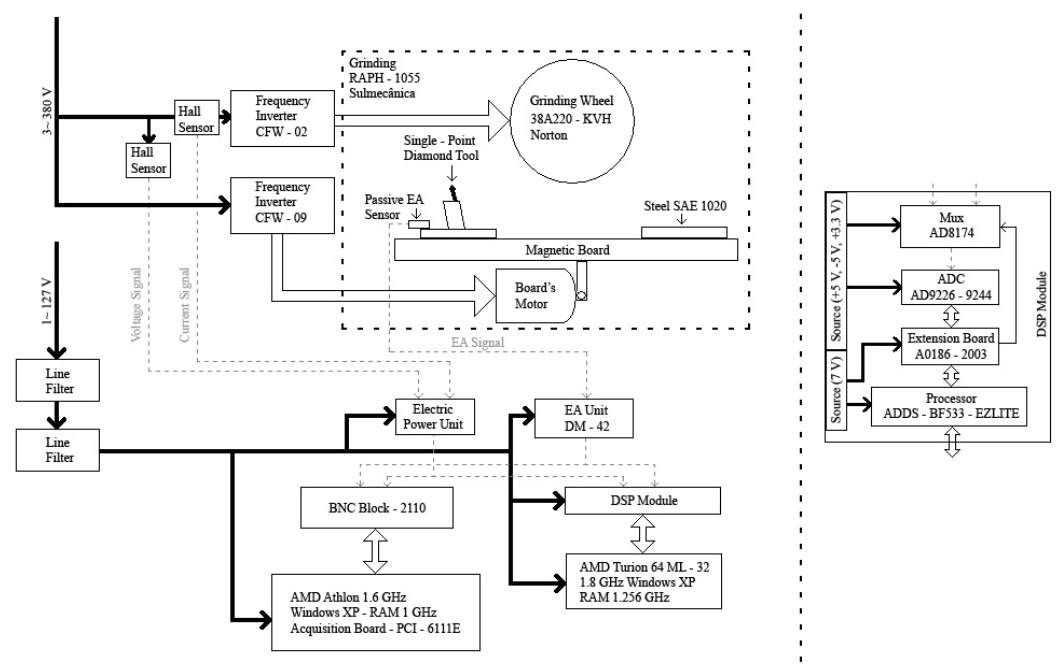

Figure 1: Schematic diagram of the test bench.

distortion in the conversion of the signal by the digital-to-analog converter used by the DSP, the acoustic emission module was adjusted to an input gain of 10 and an output gain of 20 .

The electrical power signal was captured by a power module that uses two Hall effect sensors to take voltage and current readings. The module computes the voltage and current signals, supplying the instantaneous and mean power, and is therefore interesting in the study of only the mean power.

Figure 1 presents a schematic diagram of the test bench.

The experimental part consisted of six process tests, each of which comprised six dressing passes. A different overlap factor was used in each test. Because the dressing depth value was set to a single value, the variation of the overlap factor was determined by the variation of the transverse speed of the table.

By choosing the desired overlap factor and knowing the width of the dresser's action, the rotation of the grinding table motor can be determined by (5).

The width of the dresser's action was measured with a profile projector. This procedure was carried out before each test, since the dresser undergoes wear each time it is used, causing the value bd to vary even when ad is kept constant.

$$
n=\left(\left(b_{d} \cdot 30 / U_{d}\right)+0,48\right) \cdot 30 / 0,224
$$

where $\mathrm{n}$ is the rotation parameter given in rpm which must be adjusted in the frequency inverter that controls the table's motor.

In order to produce wear on the grinding wheel before each test, several grinding passes were performed at a cutting depth of $20 \mu \mathrm{m}$ without using cutting fluid, until the burn effect occurred on a steel 1020 workpiece.

Table I lists the values of the parameters used in the tests. 
Table 1: $\quad$ Test parameters.

\begin{tabular}{|c|c|c|c|c|c|}
\hline Test & $a_{d}(\mu \mathrm{m})$ & $U_{d}$ & $b_{d}(\mu \mathrm{m})$ & $V_{d}(\mathrm{~mm} / \mathrm{s})$ & $n(\mathrm{rpm})$ \\
\hline 1st & 40,0 & 3,5 & 231,0 & 1,980 & 291 \\
\hline 2nd & 40,0 & 3,0 & 238,0 & 2,375 & 347 \\
\hline 3rd & 40,0 & 2,5 & 234,0 & 2,808 & 409 \\
\hline 4th & 40,0 & 2,0 & 233,0 & 3,498 & 507 \\
\hline 5th & 40,0 & 1,5 & 234,0 & 4,674 & 675 \\
\hline 6th & 40,0 & 1,0 & 235,0 & 7,050 & 1013 \\
\hline
\end{tabular}

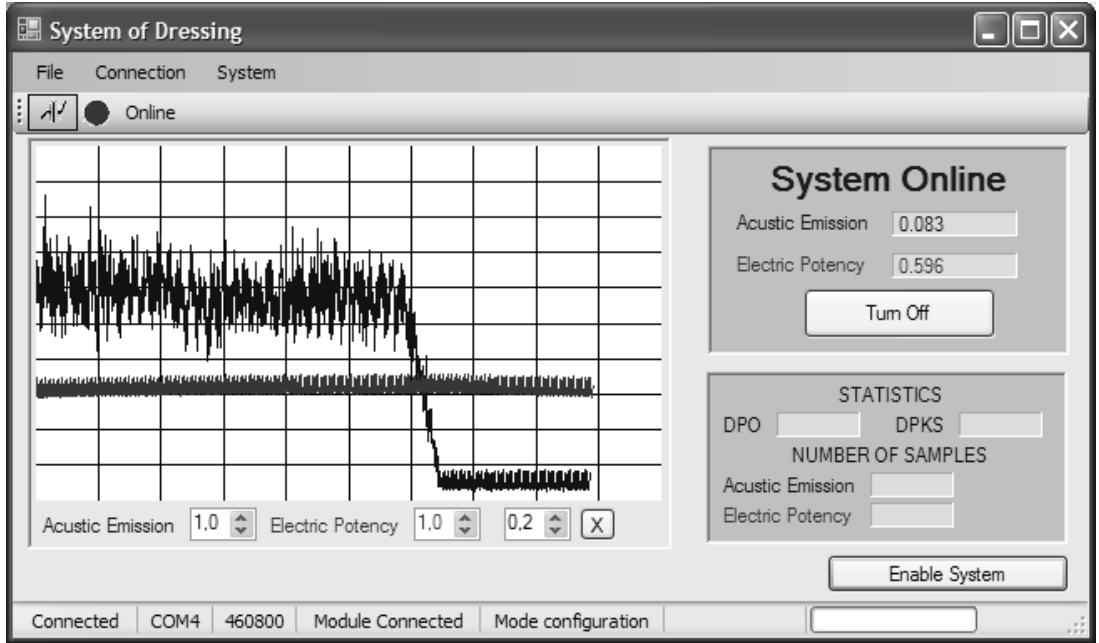

Figure 2: Online operation window with visualization of signals.

For the DSP control, a program was developed in $\mathrm{C}++$ language executed on a microcomputer. This program allows the DSP to be verified and configured, and data acquisition to be activated and deactivated.

An online visualization resource of the acoustic emission and electrical power signals monitored by the DSP was incorporated into the computer program. This resource enables one to check if the reading of the signals is occurring correctly, thereby aiding the configuration and adjustment of the system. Using this program, it is also possible to download data corresponding to the signals and statistics stored in the memory of the DSP. These data are stored in .txt type files in ASCII format. All communication between the computer and the DSP is performed serially with the RS232 protocol.

Figure 2 shows the screen of the program executing the signal visualization resource.

LabVIEW environment, in which the data acquisition board can be configured. The signals captured by the DAQ are stored by the LabVIEW application in .bin files in binary format.

To control of DAQ system, an application was used in the All the processing of the signals from the DAQ was done in the MATLAB environment. For the 
DSP system, part of the processing was performed by the device, and MATLAB was used only to calculate the mean values of the acoustic emission signal andthe electrical power. After completing all the necessary processing, the results obtained from both the systems were compared using MATLAB and Excel.

The necessary processing involves the extraction of the pass signal, filtration of the signals, and calculation of the necessary statistics.

An extraction of the pass signal involves separating from each monitored signal only the portion that corresponds to the moment when the dresser is in contact with the grinding wheel. This processing is necessary because the portion of interest for analysis is the contact between the dresser and the grinding wheel, and any data outside the pass are irrelevant.

To extract the signal, an algorithm was developed that obtains a level of reference based on samples of both the signals outside the pass and makes comparisons between new samples and the reference samples in order to determine the moment when the dresser makes contact with the grinding wheel. Because the system with DSP operates in real time, the pass extraction algorithm was executed for each new sample, and therefore only the samples referring to the pass were stored. On the other hand, for the system with DAQ operating offline, an algorithm similar to the one implemented in the DSP was executed in MATLAB, using samples inside and outside the pass.

Signal filtration processing is necessary to eliminate noise frequencies and information irrelevant to the process. Because the acquisition frequencies used here were $10 \mathrm{kHz}$ for acoustic emission and $1 \mathrm{kHz}$ for electrical power, two second-order IIR digital filters were implemented, limiting the frequency of the acoustic emission signal to $30 \mathrm{~Hz}$ and the electrical power signal to $5 \mathrm{~Hz}$, i.e., in the frequency ranges containing information relevant to the process.

After the signal extraction and filtration processing, which can be considered the preparation processing, the signals are ready for the statistical calculations, which involves calculating the values of the DPO, DPKS and the mean values of the signals. In the DSP system, the algorithm implemented in the digital signals processor performed the calculation and presented the results of the DPO and DPKS statistics, while the mean values of the signals were calculated with MATLAB software using the data obtained from the DSP.

The objective of this study was to confirm the efficiency and the possibility of implementing a DSP-based monitoring system for the dressing process, to which end a comparative analysis was made with a DAQ-based monitoring system. The algorithms used in the signals processor and in MATLAB were therefore as similar as possible and the entire processing sequence was the same, and the signals were monitored by the two systems simultaneously.

\section{Results}

Due to lack of space, only the results obtained with test number 5 are presented here. Similar results were obtained with the other tests. 

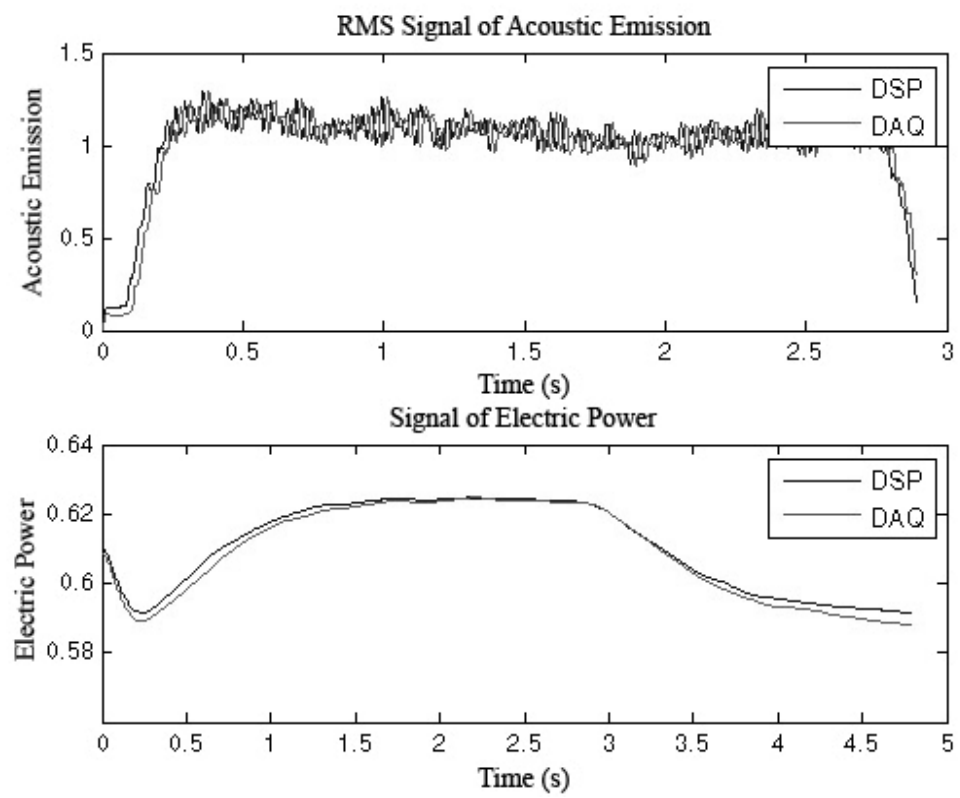

Figure 3: Curve of the fourth pass of the fifth test.

Figure 3 presents the curves of the acoustic emission and electrical power signals for pass number 4 obtained by the DSP and DAQ systems. With the help of MATLAB, the signals were superimposed to compare the quality of the data acquisition.

On these curves, the vertical scale is given in $V^{*} k$, where $k$ is a constant for the acoustic emission signal, and in $W^{*} c$, where $c$ is a constant for the electrical power signal.

An analysis of Fig. 3 reveals the similarity, in shape and behavior, of the acoustic emission signal between the DSP and DAQ systems. The predominant differences in the acoustic emission signal are the time deviation and the out of pass value that occur in the DAQ data.

The first difference is justified by the method used in extracting the pass. Because the data that are relevant for processing are only those that occur during the contact between dresser and grinding wheel, the other data must be excluded. It should be kept in mind that, for the detection and extraction of the pass, the samples of signals out of pass are used as references and the data acquisition hardware of the DSP and DAQ systems is different. Therefore, there may be differences in the sampled values, which may lead to the deviation depicted in Fig. 3.

One way to reduce this deviation is by means of a fine adjustment of the parameters of the A/D converters of both systems. However, since this difference was small and what is relevant in the processing is the behavior of the signal, the intensity of the deviation is acceptable. 
In Fig. 3, note that the electrical power signal shows a greater difference, which was more marked in the first tests, whose data could not be presented here due to lack of space.

With regard to the power signal, it is important to point out the high noise load from the electrical installation of the test site. This problem prevented the use of the power pass extraction algorithm, and the acoustic emission signal therefore had to be used as time reference for the pass extraction of the electrical power signal.

Another important factor to be considered for the Power signal is the sampling rate, which was different on the two systems. The DSP used different sampling frequencies, which were $10 \mathrm{kHz}$ for acoustic emission and $1 \mathrm{kHz}$ for power, while the DAQ, due to a hardware characteristic, shares the same frequency between the two channels, thus precluding the use of different rates. Thus, the reading speed of power with the DAQ was 10 times that used in the DSP, generating a larger volume of points and leading to the need for a reduction by the software in order to equalize the number of points and the sampling rate. This process of reduction was performed with MATLAB before the pass extraction algorithm.

Moreover, analyzing the signals in Fig. 4c and Fig. 4d, one can see that the mean acoustic emission signal varied significantly, while the mean electrical power signal was practically constant throughout the tests. This minor variation in power is related to the dimensions of the grinding wheel and the dresser.

Because the dresser used here was a single-tip diamond with a cutting depth of $40 \mu \mathrm{m}$, whose width of action is between 231 and $238 \mu \mathrm{m}$, these dimensions are negligible when compared to the diameter of the grinding wheel. Hence, in these conditions, the passage of the dresser across the grinding wheel does not require significant additional power from the motor.

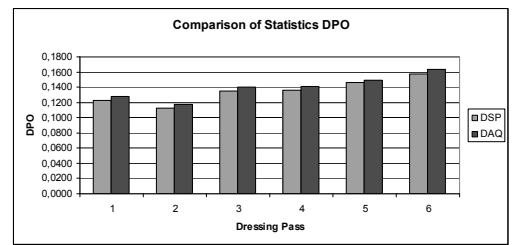

(a)

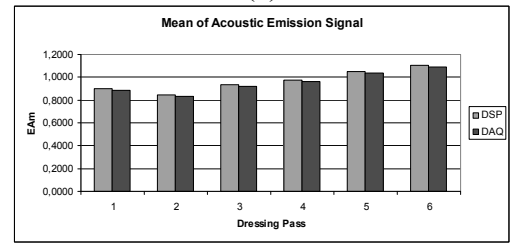

(c)

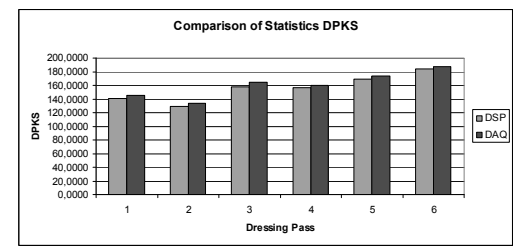

(b)

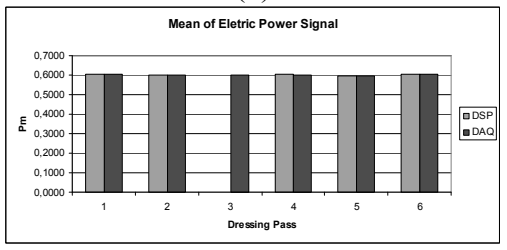

(d)

Figure 4: Fifth text. (a) Comparison of statistic DPO. (b) Comparison of statistic DPKS. (c) Comparison of the mean of acoustic emission signal. (d) Comparison of the mean of electric power signal. 
Therefore, based on this consideration and analyzing all the tests, it can be proposed that the signal of interest in monitoring the dressing process is the acoustic emission signal.

An examination of Fig. 4a, Fig. 4b and Fig. 4c reveals similarities in the behavior of the mean acoustic emission signal and the DPO and DPKS parameters. One can also see that the value of these three statistics shows the same behavior in the two systems used here, with a minor error of value. This similarity was observed in all the tests.

In view of the fact that, to determine the end of the dressing process, one must observe the behavior of the DPO and DPKS statistics, which should tend to stabilize at a constant value, we can propose the substitution of the calculation of the DPO and DPKS parameters for a calculation of the mean acoustic emission signal, thus simplifying the calculations and reducing the computational effort. This proposal is possible due to the similarity of the behavior of the DPO and DPKS statistics and that of the mean acoustic emission. The behavior obtained from the statistics was as expected, i.e., their increase along with increasing passes. For the test presented in Fig. 3 and Fig 4, it can be seen that starting from the fourth pass, the value assumes a practically constant level, indicating that the process can be concluded. However, we cannot generalize and state that the grinding wheel is dressed with four passes, since the number of passes required depends on the initial conditions of the grinding wheel, the type of dresser, the dressing depth, and the table's speed.

\section{Conclusion}

Based on the results obtained and according to the analyses performed, it can be inferred that the use of a hardware system with a digital signal processor operating in real time mode is possible and viable from the practical standpoint, and that specific hardware can be developed to monitor the dressing process.

The main factor observed was the data acquisition capacity through the DSP without the occurrence of signal losses or distortions. After the tests and analysis of the results, it was found that the DSP hardware DSP has sufficient speed for operations in real time, without causing loss or distortion of signals.

Because the errors presented were minor, never exceeding $10 \%$, they can be considered acceptable, considering that they can be reduced by making finer hardware adjustments.

The analysis of the results indicates that only the acoustic emission signal requires monitoring. Moreover, calculation of the DPO and DPKS parameters can be substituted for calculation of the mean effective acoustic emission signal.

Lastly, according to this study, the use of DSP on the production line and, in principle, in the dressing process, is commercially feasible and can constitute a new tool to aid this very important fabrication stage in industry, improving the product's final quality, and hence, adding value to the product. 


\section{References}

[1] J.F.G. Oliveira, E.J. Silva, M. Biffi, \& F. Matrai, "New architecture control system for an intelligent high speed grinder," Abrasives Magazine, 2002, pp. 4-11.

[2] A. Hassui, A.E. Diniz, J.F.G. Oliveira, J. Felipe, \& J.J.F. Gomes, "Experimental evaluation on grinding wheel wear through vibration and acoustic emission," Wear, vol. 217, 1998, pp. 7-14.

[3] J.A. Badger \& A. Torrance, "Burn awareness-Understanding the causes of grinding burn helps alleviate the problem," Cutting Tool Engineering Magazine, vol. 52, 2000, p. 12.

[4] B. Karpuschewski, M. Wehmeier, \& I. Inasaki, "Grinding Monitoring System Based on Power and Acoustic Emission Sensors," CIRP Annals Manufacturing Technology, vol. 49, 2000, pp. 235-240.

[5] J. Webster, W.P. Dong, \& R. Lindsay, "Raw acoustic emission signal analysis of grinding process," CIRP Annals-Manufacturing Technology, vol. 45, 1996, pp. 335-340.

[6] P.R. Aguiar, E.C. Bianchi, \& J.F.G. Oliveira, "A method for burning detection in grinding process using acoustic emission and effective electrical power signal," CIRP Journal of Manufacturing Systems, Paris, vol. 31, 2002, pp. 253-257.

[7] F.R.L. Dotto, P.R. Aguiar, E.C. Bianchi, P.J.A. Serni, \& R. Thomazella, "Automatic system for thermal damage detection in manufacturing process with internet monitoring," Journal of the Brazilian Society of Mechanical Sciences and Engineering, vol. 28, 2006, pp. 153-160.

[8] C.F.L.P. Hellmeister, "Monitoramento da Dresagem na Retificação através do Sinal Puro de Emissão Acústica," Mestrado, Universidade Estadual Paulista, 2004.

[9] P.R. Aguiar, A.G. Souza, E.C. Bianchi, R.R. Leite, \& F.R.L. Dotto, "Monitoring the dressing operation in the grinding process," International Journal of Machining and Machinability of Materials , vol. 5, 2009, pp. 3 22.

[10] S. Malkin, Grinding Technology: Theory and Applications of Machining with Abrasives, Dearborn: SME, 1989.

[11] S. Sultana, "Effect of dressing parameters on grinding wheel wear," Mestrado, Dalhousie University, 2004.

[12] I.D. Marinescu, W.B. Rowe, B. Dimitrov, \& I. Inasaki, Tribology of Abrasive Machining Processes, Norwich, NY: William Andrew, 2004.

[13] S.C. Salmon, Modern Grinding Process Technology, New York: McGrawHill, 1992.

[14] J.F.G. Oliveira, D.A. Dornfeld, \& B. Winter, "Dimensional characterisation of grinding wheel surface through acoustic emission," CIRP Annals, vol. 43, 1994, pp.291-294.

[15] I. Inasaki, "Monitoring of dressing and grinding process with acoustic emission signals," CIRP Annals, vol. 34, 1985, pp.277-280. 
[16] J. Webster, I. Marinescu, \& R. Bennett, "Acoustic emission for process control and monitoring of surface integrity during grinding," CIRP Annals, vol. 43, 1994, pp.299-304.

[17] W. Konig, \& H.P. Meyen, "AE in grinding and dressing: accuracy and process reliability," Proc. of 4th Int. Grind. Conference, 1990, MR90-526.

[18] J. Kwak, \& M. Ha, "Neural network approach for diagnosis of grinding operation by acoustic emission and power signals," Journal of Materials Processing Technology, vol. 147, 2004, pp.65-71.

[19] D.E. Lee, I. Hwang, C.M.O Valente, J.F.G. Oliveira, \& D.A. Dornfeld, "Precision manufacturing process monitoring with acoustic emission," International Journal of Machine Tools and Manufacture, vol. 46, 2006, pp.176-188.

[20] B. Linke, "Dressing process model for vitrified bonded grinding wheels," CIRP Annals - Manufacturing Technology, vol. 57, 2008, pp. 345-348.

[21] H.T. Young, \& D.J. Chen, "Online dressing of profile grinding wheels," Int. J. Adv. Manuf. Technol., vol. 27, 2006, pp. 883-888.

[22] Li Xue, F. Naghdy, \& C. Cook, "Monitoring of wheel dressing operations for precision grinding," Industrial Technology, 2002. IEEE ICIT '02. 2002 IEEE International Conference on, vol. 2, 2002, pp 1296-1299. 